\title{
An Enhanced Millimeter-Wave Foliage Propagation Model
}

\author{
Feinian Wang, Student Member, IEEE, and Kamal Sarabandi, Fellow, IEEE
}

\begin{abstract}
In this paper, the behavior of wave propagation through coniferous forest stands at millimeter-wave frequencies is characterized both theoretically and experimentally. A coherent wave propagation model is used to simulate the propagation through foliage. The coherent model is composed of two components: a forest stand generator that makes use of a stochastic fractal model, and an electromagnetic model that makes use of Foldy's approximation and single scattering. An outdoor measurement system is designed and used for characterizing the channel behavior for a pine tree stand at $K a$-band $(35 \mathrm{GHz})$. In this experiment, 84 independent spatial samples of transmitted signal through the pine stand were collected to obtain the path-loss statistics. The comparison between measurement and simulation results showed that single scattering theory overestimates the wave attenuation through foliage. To improve the accuracy of the coherent model, partial multiple scattering occurred among the needles of highly dense leaf clusters must be included for the estimation of the coherent attenuation. Distorted Born approximation is used to macromodel the scattering pattern from needle clusters. This technique has comparable accuracy and requires much less computational resources than a full-wave solution, such as method of moment. By including multiple scattering effects of needle clusters in the simulation model, much better agreement is obtained for both mean and standard deviation of the path-loss.
\end{abstract}

Index Terms-Distorted Born approximation (DBA), multiple scattering, path-loss, propagation model.

\section{INTRODUCTION}

$\mathbf{T}$ HE DEMAND for high data rate communication is on the rise. For this purpose wide-band communication at millimeter-wave frequencies is under consideration for a significant number of applications. Forested environment poses a significant challenge for the operation of such systems. In order to assess the performance of communication devices operating at high frequencies, characteristics of the communication channel such as path-loss, coherence bandwidth, fading statistics, etc., must be determined. Wave propagation behavior in forested environment is also of interest for the problem of foliage-camouflaged target detection. To predict the wave propagation channel characteristics, an accurate electromagnetic model for forested environment is required.

In recent years physics-based models using either radiative transfer theory (incoherent approach) or wave theory (coherent

Manuscript received September 2, 2003; revised June 3, 2004. This work was supported in part by the DARPA FCS Communications Project under Subcontract S01-19 from the Pennsylvania State University and in part by the DARPA MURI Project 43584-EV-MUR.

The authors are with the Radiation Laboratory, Department of Electrical Engineering and Computer Science, The University of Michigan, Ann Arbor, MI 48109 USA (e-mail: saraband@eecs.umich.edu).

Digital Object Identifier 10.1109/TAP.2005.850704 approach) have attained significant prominence [1]. The wave theory model is believed to be more accurate for presenting the coherence effects and phase information. In [2] and [3], coherent wave propagation models based on Monte Carlo simulation of scattering from a realistic looking fractal trees are used to obtain the statistics of wave propagation through foliage successfully. The tree stands were generated with physical and structural parameters, such as tree density, height, mean trunk diameter, etc., from the ground truth measurements. The randomness of the forest medium was accounted for through a Monte Carlo simulation. Scatterers, such as branches and leaves, inside the forest are illuminated by the coherent mean-field, and the scattered fields are added coherently. Both the illuminating mean-field and the scattered fields are attenuated by the forest medium. To simplify the model and to reduce the computational complexity, multiple scattering among branches and leaves was ignored. Such approximation is reasonable for the frequency bands where multiple scattering effects are negligible. However, at upper microwave and millimeter-wave frequencies, where the size of densely packed particles becomes larger or comparable with the wavelength, the single scattering model is not sufficient. Multiple scattering effect is manifested in both mean-field and incoherent scattered power. Basically near-field multiple scattering, such as those happened within a dense leaf cluster, influences the value of forward scattering amplitude which determines the attenuation rate of the coherent mean-field through the foliage. Likewise, the incoherent scattered power generated from scatterers is affected by the multiple scattering between scatterers.

The multiple scattering occurred among leaves of highly dense leaf clusters is included to improve the accuracy of the wave propagation model. Other constituents of the forest, such as branches and trunks, however are distributed sparsely and single scattering is still applicable for these scatterers. The widely used Foldy's approximation [4] in conjunction with single scattering theory is shown to overestimate the attenuation rate at millimeter-wave frequencies [5]. In [5] a full-wave numerical technique, method of moment (MoM) [6], is used to calculate the scattering from a cluster of leaves or needles exactly. However, this technique requires significant computational resources which prohibit its direct application in the wave propagation model. In this paper, a computationally efficient technique is presented that accounts for the multiple scattering in needle clusters. For a semi-random cluster of pine needles the scattered field remains coherent only in the forward direction and a small angular range near the forward direction, which depends on the size of the cluster relative to the wavelength. To calculate the coherent field, distorted 


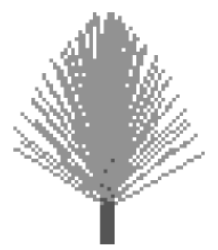

(a)

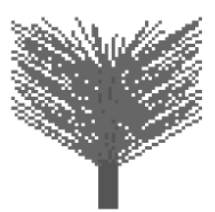

(b)
Fig. 1. Needle cluster structures: (a) end-cluster and (b) stem-cluster.

Born approximation (DBA) is applied to an inhomogeneous, anisotropic dielectric object having the exact boundary as the needle cluster. The scattered field outside the forward scattering cone has a random phase with almost uniform scattered power pattern. In situations where a clear boundary between leaf clusters and the surrounding air cannot be recognized, such as those of deciduous trees, this technique is not applicable. However, for those sparse leaf clusters, the effect of multiple scattering is not as significant.

To examine the accuracy of the wave propagation model, an outdoor measurement through a pine tree stand was conducted at $\mathrm{Ka}$-band $(35 \mathrm{GHz})$. Simulation results using single scattering model and multiple scattering model are compared with the measured result, and the importance of the latter is clearly justified.

In what follows, we first investigate the multiple scattering effects from realistic pine needle clusters. Next, the macromodel describing the scattering from the clusters is described, then the outdoor measurement procedure is presented in detail. Finally the measurement and simulation results are compared.

\section{Multiple Scattering EfFects From NeEdle Clusters}

In this section, a MoM solution for the calculation of the scattered field from red pine needle clusters is presented and compared with single scattering solution to investigate the multiple scattering effects. The computational requirements are also examined to justify the need for developing a macromodel for the cluster. Since the macromodel encapsulates the effect of many needles $(\sim 100)$, the computation time for the propagation model is reduced.

A red pine needle cluster has its needle buds distributed as three concentric spirals around a small stem. Needles come off of the stem at an angle which will be referred to as the tilt angle. There are two kinds of clusters on coniferous trees, the endcluster and the stem-cluster. For the end-cluster, the tilt angle decreases as the needle bud approaches the tip of the stem. For the stem-cluster, the tilt angles are the same for each needle. The distance between each pair of needles can be as small as $5 \mathrm{~mm}$, less than half a wavelength at $35 \mathrm{GHz}$, and the needle length can vary from 1 to $10 \mathrm{~cm}$, which is much larger than a wavelength. In this case multiple scattering among needles may be significant. Based on a ground truth measurement for the red pine stand used in the path-loss measurement, the end-cluster and the stemcluster (see Fig. 1) were measured to have an average of 96 and 117 needles, respectively. The average needle length and diameter for this pine tree were measured to be 35 and $0.45 \mathrm{~mm}$, respectively.

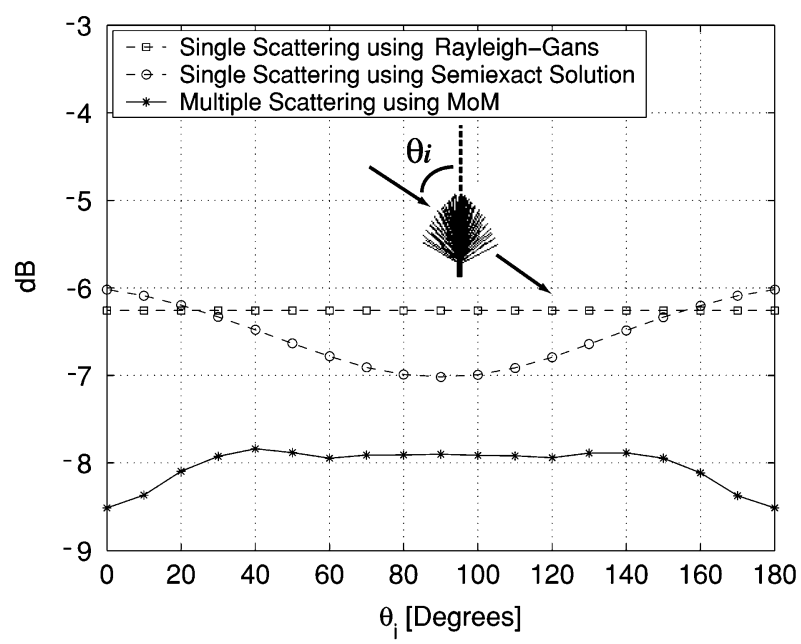

(a)

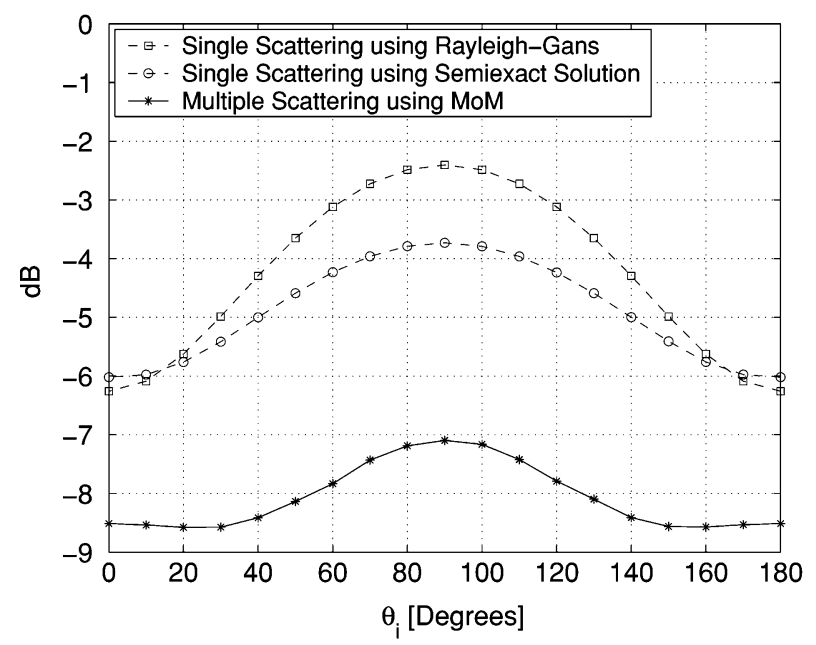

(b)

Fig. 2. Comparison of forward scattering from an end-cluster: (a) $\left|S_{h h}\right|$ and (b) $\left|S_{v v}\right|$.

As in [5], MoM is applied to examine the multiple scattering from each cluster. For comparison, the single scattering from needle clusters is also calculated where the scattered field from each single needle is added coherently. Two solutions are employed to compute the scattered field from a single needle. One is based on a low frequency technique, Rayleigh-Gans approximation [7], which is quite simple but not accurate at millimeterwave frequencies. The other one is the semi-exact solution based on the eigen series solution for scattering from an infinite dielectric cylinder [8] which is accurate but more complicated in computation. Fig. 2 shows the forward scattering from the end-cluster versus the incident angle $\theta_{i}$ (angle between the stem axis and the incident wave). It is obvious that the Rayleigh-Gans approximation is no longer valid at millimeter-wave frequencies, and the semi-exact single scattering solution overestimates the forward scattering by an amount as large as $3 \mathrm{~dB}$, compared with the multiple scattering solution using MoM. Same phenomenon is observed for stem-cluster case.

According to Foldy's approximation, the attenuation rate is proportional to the imaginary part of the forward scattering amplitude. Examination of such quantity based on the above 
simulation shows that using single scattering theory to calculate scattering from needle clusters will cause a significant overestimation of the attenuation rate which mandates inclusion of the effect of multiple scattering. That is, if the effects of multiple scattering is ignored, calculation of the mean-field through the foliage will be erroneous. On the other hand, it is quite challenging to keep track of multiple scattering in needle clusters, as MoM is computationally intensive. First, according to the MoM algorithm described in [5], direct inversion of the impedance matrix is required in order to reuse it for clusters with same structure but different orientations. This poses great challenges on the computer memory and speed requirements. For example, at $35 \mathrm{GHz}$, a typical end-cluster shown in Fig. 1 requires about $387 \mathrm{MB}$ memory to store the impedance matrix, and a stem-cluster requires $471 \mathrm{MB}$. If the number or length of needles increases, the number of cells (or unknowns) will also increase. To account for variabilities in clusters many of such matrices must be stored. Moreover, since the direct inversion algorithm must be employed and the computation time is proportional to $N^{3}$, where $N$ is the number of unknowns, the time for inverting the impedance matrices could be prohibitively long. For instance, it takes about 1 hour to invert a matrix with 6000 unknowns using a Linux machine with $2.4 \mathrm{GHz}$ processor.

Second, even when the inverse impedance matrices are stored for reuse, the direct usage of the pre-stored matrices is still prohibitive since there are many clusters in one tree, many trees in a forest, and many realizations required by the Monte Carlo simulation. A better approach is to calculate and pre-store the full bistatic scattering matrix, $S_{m n}\left(\theta_{i}, \phi_{i}, \theta_{s}, \phi_{s}\right)$, where $m, n$ stand for vertical or horizontal polarization, $\theta_{i}, \phi_{i}$, and $\theta_{s}, \phi_{s}$ represent the incident and scattering angles, respectively. The pre-stored $\overline{\bar{S}}$ can be used as a lookup table which can be searched based on the incident and scattering angles. However to generate $\overline{\bar{S}}$ lookup table for each cluster, more than $1 \mathrm{~GB}$ of memory and $3 \mathrm{~h}$ of computation time are required. Our goal is to find an alternative approach to the aforementioned techniques. Basically a macromodel based on an analytical method and computational results obtained from the MoM is proposed.

\section{Macromodeling of Multiple Scattering From NeEDle Clusters}

In this section, several statistical behavior of scattered field important to the development of the macromodel are presented first and then the detailed algorithm using DBA to model scattering near forward direction is described. Finally the comparison between the macromodel results and those obtained from the brute force full-wave numerical simulations are presented.

\section{A. Statistical Behavior of Scattering From Needle Clusters}

In this section the statistical behavior of the scattered field from needle clusters are studied. The key features of the statistical behavior of the scattered field are exploited in the derivation of a simple macromodel.

Outside the forward scattering beam, the scattered field is incoherent and its level is substantially lower than that in the forward direction. It is also observed that the average power

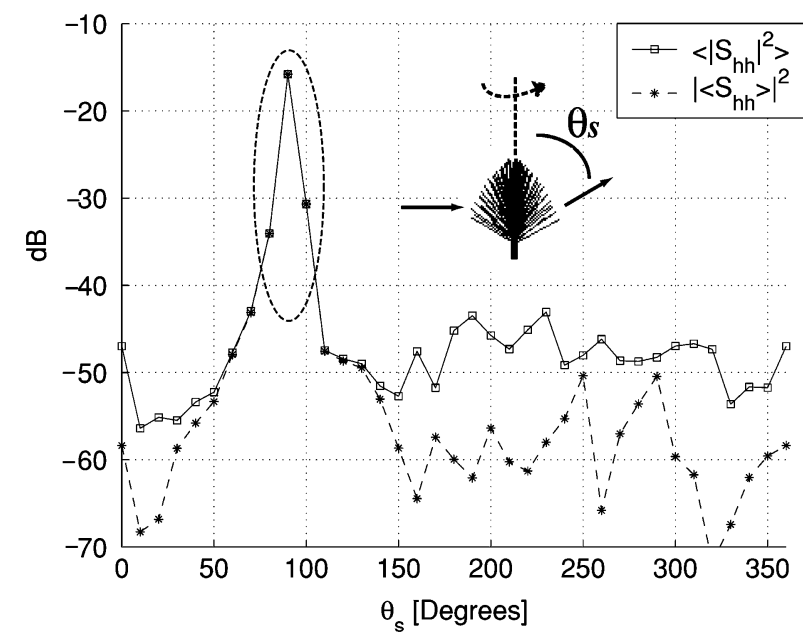

Fig. 3. Bistatic scattering from a needle cluster, averaged over the rotation angle around central stem (H-polarization).

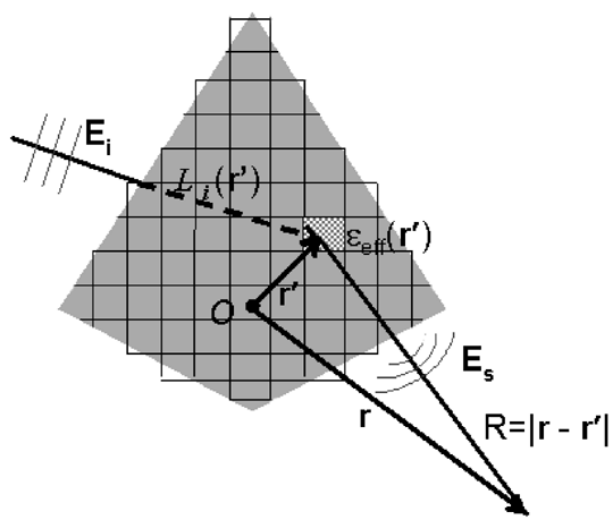

Fig. 4. Algorithm of DBA.

of bistatic scattering from needle cluster outside the forward scattering cone region is almost constant. That is, the scattered power can be modeled as a constant and the field can be given a random phase. The following scattering simulations demonstrate these assertions.

For a needle cluster inside a forest canopy, the rotation angle around its central stem is random. Also position, pitch angle, and length of needles on a stem has certain degree of randomness. These random parameters are used in a Monte Carlo simulation of bistatic scattering from needle clusters to study the statistical behavior of the bistatic pattern. Fig. 3 shows the H-polarization bistatic scattering pattern of a needle cluster having 96 needles of diameter $0.45 \mathrm{~mm}$, length $3.54 \mathrm{~cm}$, and relative dielectric constant $6.6+j 6.2$ at $35 \mathrm{GHz}$. Similar pattern is obtained for $\mathrm{V}$-polarization case. The solid line represents the average scattered power and the dashed line represents the power of the averaged field. The significant difference between the power of the mean-field and total power outside the forward scattering beam indicates that the phase of the scattered field along these directions varies randomly, that is, the field is totally incoherent. In addition, the averaged power has relatively small fluctuation. Therefore, a constant function can be used to model average scattered power over the scattering angles outside forward scattering beam. The scattered field in this region can be given a random phase. 


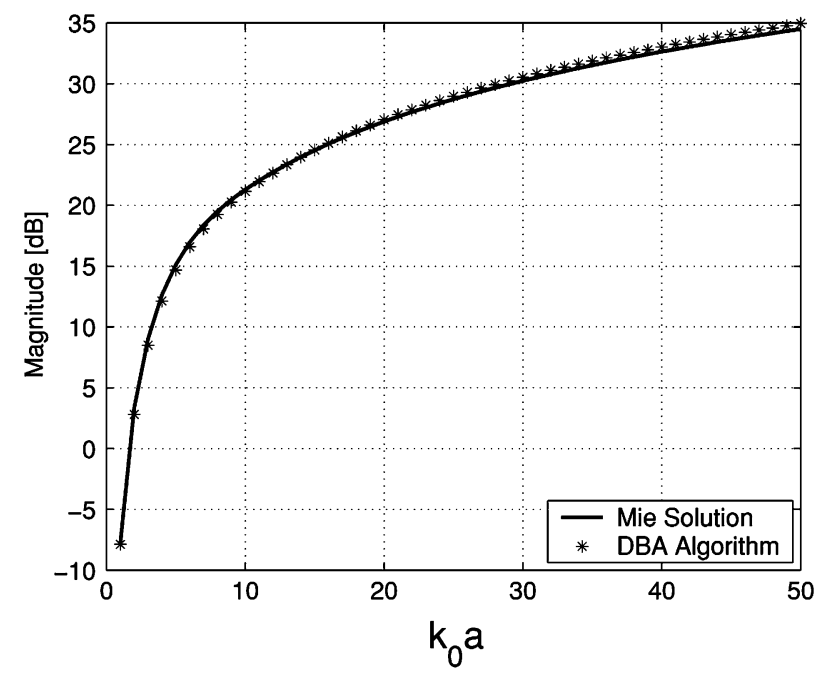

(a)

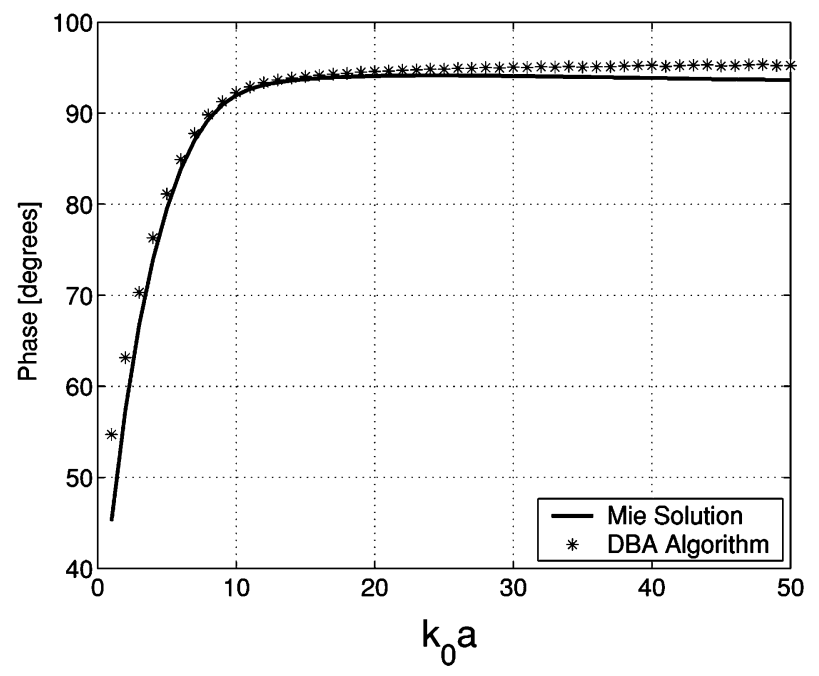

(b)

Fig. 5. Forward scattering from a dielectric sphere $\left(\epsilon_{r}=1.5+j 0.5\right)$ versus $k_{0} a$, computed by DBA algorithm and Mie solution: (a) magnitude (in decibels) and (b) phase (in degrees).

Near the forward direction, scattering from all individual scatters are in-phase and therefore the mean-field is strong only in this region. Over the forward scattering beam the total scattered power and the power of the mean-field are identical. This indicates that over this region the scattering is coherent. The accuracy requirement of macromodel in this region is much more strict since the attenuation rate is directly proportional to the imaginary part of the forward scattering amplitude. To model the mean-field it is sufficient to model scattering from the "average" scatterer. The average scatterer can be viewed as a body of revolution having a surface defined by the envelope of the needles tip as shown in Fig. 4. Due to preferred vertical orientation of needles in the cluster and the variation of vegetation volume fraction within the cluster volume, the average scatterer is modeled by an inhomogeneous anisotropic medium. As an initial estimate, using a simple mixing formula [9], the average permittivity of the end and stem-clusters at $35 \mathrm{GHz}$ are calculated to be around $1.16+j 0.18$ and $1.086+j 0.095$, respectively. This effective dielectric constant is not low enough to allow for the application of Born approximation in the calculation of bistatic scattered field. However, accounting for the attenuation and phase velocity of the incident wave through the effective dielectric block, i.e., using DBA, the interior field can be estimated and used to calculate the scattered field. In the next section, we will examine the region of validity of DBA method.

\section{B. $D B A$}

In this section, a general algorithm for calculation of scattering from an arbitrary dielectric object is described first, then the validity region of such method is examined by comparing the exact solution for a dielectric sphere with that obtained from DBA. Next, the DBA algorithm is applied to the needle clusters and the simulation results are compared with the MoM solution.

1) DBA Algorithm: DBA (or Rytov approximation [10]) is a classic method for computation of scattered field from dielectric objects whose permittivity is close to that of the surrounding medium. The first step of the proposed DBA algorithm is target discretization and phase change of incident field as shown in Fig. 4. The interior field of the cell centered at the point $\overline{r^{\prime}}, \bar{E}\left(\overline{r^{\prime}}\right)$, is computed by calculating attenuation and phase change along the path $L_{i}\left(\bar{r}^{\prime}\right)$. The induced polarization currents in each cell is then easily obtained from $\bar{J}_{p}\left(\overline{r^{\prime}}\right)=$ $-i k_{0} Y_{0}(\epsilon-1) \bar{E}\left(\overline{r^{\prime}}\right)$. In this approximation the interior field of the dielectric object is approximated by a field similar to the incident field having the same amplitude and polarization but a different propagation constant. The propagation constant of this field is that intrinsic to the medium the scatterer is made of. Because of its simplicity, DBA can be applied to scatterers of arbitrary shape and dielectric profile. The scattered field in the far-field region at an observation point $\bar{r}$ is then given by

$$
\begin{aligned}
\bar{E}_{s}(\bar{r})= & j \omega \mu_{0} \iint_{V} \int \overline{\bar{G}}\left(\bar{r}, \bar{r}^{\prime}\right) \cdot \bar{J}_{p}\left(\bar{r}^{\prime}\right) d V^{\prime} \\
= & \frac{k_{0}^{2}}{\pi} \iint_{V} \int \frac{e^{j k_{0} R}}{R}\left(\overline{\bar{I}}-\hat{k}_{s} \hat{k}_{s}\right) \cdot\left(\epsilon_{\mathrm{eff}}^{=}\left(\bar{r}^{\prime}\right)-\overline{\bar{I}}\right) \\
& \cdot \bar{E}\left(\bar{r}^{\prime}\right) e^{-j k_{0} \hat{k}_{s} \cdot \bar{r}^{\prime}} d V^{\prime}
\end{aligned}
$$

where $\overline{\bar{G}}$ is the free space dyadic Green's function, $R$ is the distance from the cell center to the observation point, $\hat{k}_{s}$ is the unit vector along the scattering direction, and $\overline{\bar{I}}$ is the dyadic idemfactor. $\overline{\overline{\epsilon_{\mathrm{eff}}}}\left(\bar{r}^{\prime}\right)$ represents the effective permittivity tensor of the medium. The attenuation and phase change of the incident field along the path $L_{i}\left(\bar{r}^{\prime}\right)$ can be simply calculated by identifying cells along this path.

2) Validity Region of DBA: Although DBA is a classic approach, except qualitatively, its region of validity in terms of dielectric contrast and object size is not reported in the literature.

The exact Mie series solution of the scattering from a dielectric sphere is well-known. Fig. 5(a) and (b) show the magnitude and phase of the forward scattering as function of normalized size $k_{0} a$ ( $k_{0}$ is the free space wavenumber, $a$ is the radius of the sphere), computed by the DBA algorithm and Mie solution, 


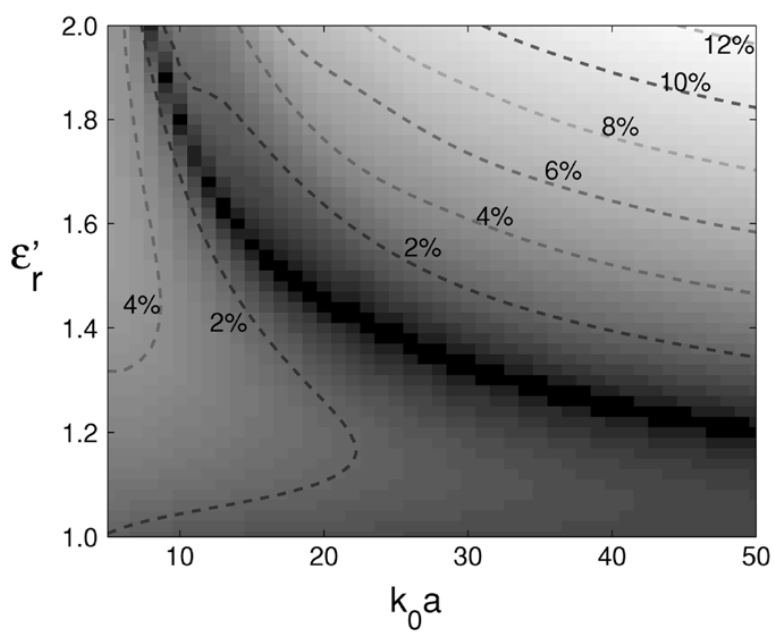

(a)

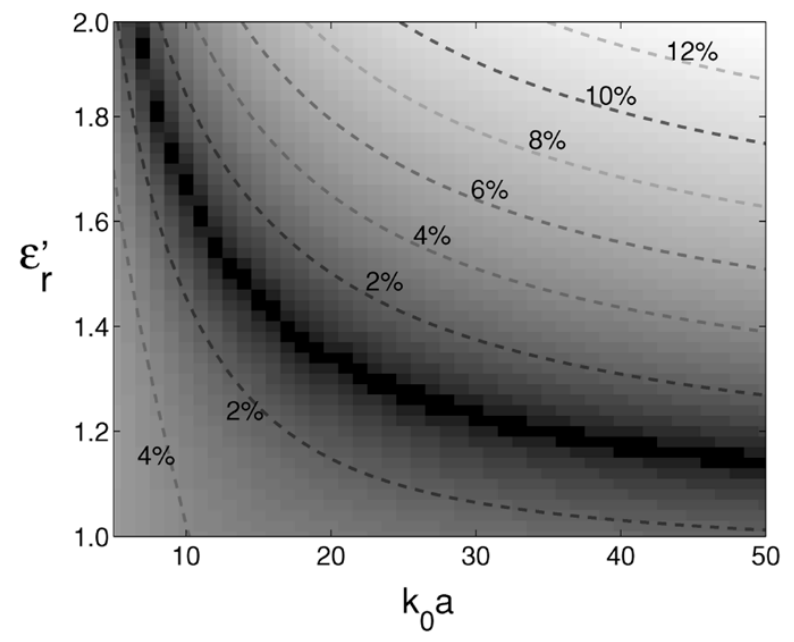

(b)

Fig. 6. Percentage error of forward scattering magnitude computed by DBA algorithm compared to Mie solution for a dielectric sphere with fixed loss tangent, versus normalized size $k_{0} a$ and real part of relative dielectric constant $\epsilon_{r}^{\prime}$ : (a) loss tangent $=0.1$ and (b) loss tangent $=0.3$.

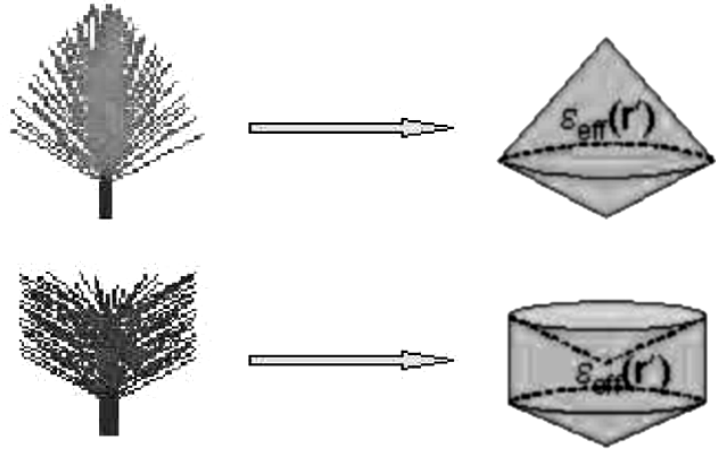

Fig. 7. Effective dielectric blocks approximated from needle clusters.

for a dielectric sphere with $\epsilon_{r}=1.5+j 0.5$ at $35 \mathrm{GHz}$. A very good agreement is shown for spheres as large as $k_{0} a=50$. This agreement verifies correct implementation of DBA and indicates that DBA algorithm can be applied to needle clusters at millimeter-wave frequencies. Fig. 6(a) and (b) show the percentage errors of the magnitude of the forward scattering versus the normalized size $\left(k_{0} a\right)$ and the real part of the relative dielectric constant $\left(\epsilon_{r}^{\prime}\right)$ assuming a fixed loss tangent $(\tan \delta)$ as 0.1 and 0.3 , respectively. Less than $10 \%$, i.e., $1 \mathrm{~dB}$ error for magnitude is achieved for $k_{0} a$ up to 50 and $\epsilon_{r}^{\prime}$ up to 2.0 in both cases. Phase error (not shown in Fig. 6) of less than 3\% is achieved as well. It is observed from Fig. 6(a) and (b) that a null (minimum error) exists in both cases, and above the null, the error increases as $k_{0} a$ and $\epsilon_{r}^{\prime}$ increase. The larger the loss tangent, the closer the null is to the bottom-left corner of the figure, and the larger error for the same $k_{0} a$ and $\epsilon_{r}^{\prime}$ above the null.

3) Scattering From Needle Clusters Using DBA: As mentioned earlier needle clusters cannot simply be modeled by homogeneous isotropic medium. Fig. 7 shows the surfaces of "the average scatterer" for an end-cluster and a stem-cluster. The end-cluster is approximately a double-cone and the stem-cluster resembles a cylinder with concave and convex conical ends. The effective permittivity profile is computed according to the local needle volume fraction. For instance, in

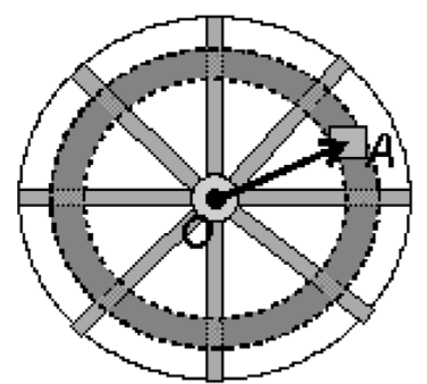

(a)

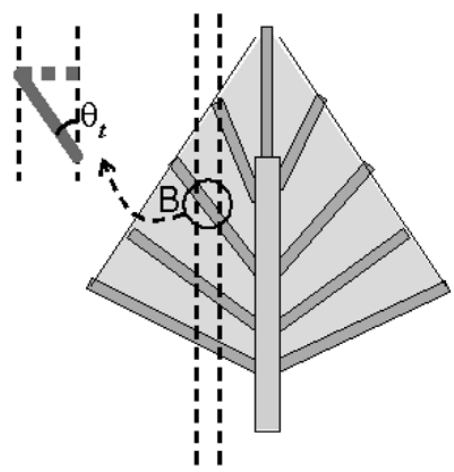

(b)
Fig. 8. (a) Transverse slice of the needle clusters and (b) longitudinal slice of the end-cluster.

both end-cluster and stem-cluster, the needle volume fraction decreases radially. Fig. 8(a) shows a transverse slice of a needle cluster. The needle volume fraction is inversely proportional to radial distance $|O A|$. For end-clusters, the needle volume fraction also increases from the bottom to the tip due to the decrease of the tilt angle of the needles. Fig. 8(b) shows a longitudinal slice of an end-cluster. The needle volume fraction is proportional to $1 / \sin \theta_{t}$, where $\theta_{t}$ is the local needle tilt angle.

Once the information related to needle volume fraction is known, the effective permittivity at each point can be calculated using Clausius-Mossotti dielectric mixing formula [11]

$$
\overline{\overline{\epsilon_{\mathrm{eff}}}}=\epsilon_{h}\left[\overline{\bar{I}}+\left(\overline{\bar{I}}-\frac{\eta\langle\overline{\bar{\alpha}}\rangle}{3 \epsilon_{h}}\right)^{-1} \cdot \frac{\eta\langle\overline{\bar{\alpha}}\rangle}{\epsilon_{h}}\right]
$$

where $\epsilon_{h}$ is the permittivity of the background medium which is air, $\eta$ is the needle volume fraction of the cell, and $\langle\overline{\bar{\alpha}}>$ is the polarizability tensor averaged over the needle orientation angle. For a needle segment oriented at angle $\left(\theta_{t}, \phi_{t}\right)$ the associated polarizability tensor of the needle can be calculated as

$$
\overline{\bar{\alpha}}=\overline{\bar{T}}^{-1} \cdot \overline{\overline{\alpha_{0}}} \cdot \overline{\bar{T}}
$$



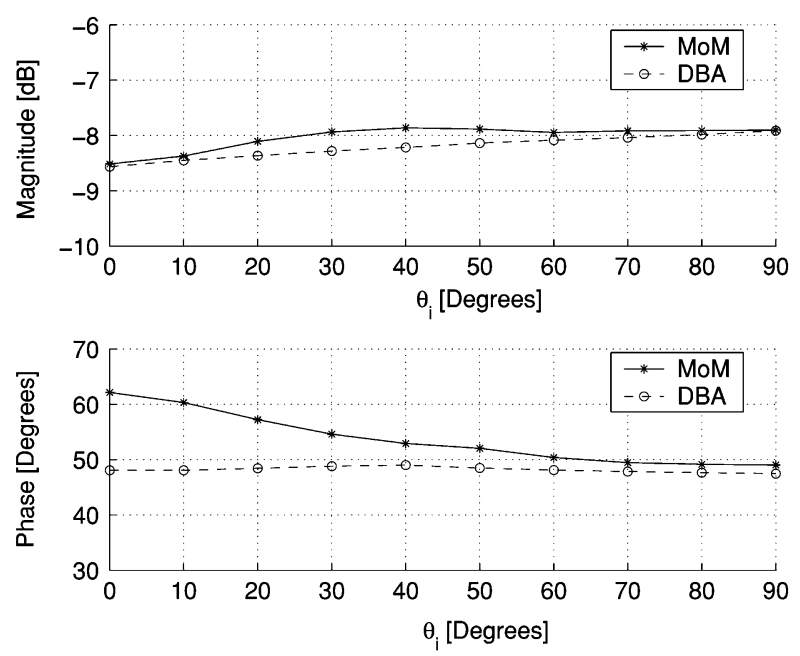

Fig. 9. Comparison of forward scattering from a needle cluster between DBA and MoM: $S_{h h}$.

where, $\overline{\overline{\alpha_{0}}}$ is the polarizability tensor when $\left(\theta_{t}, \phi_{t}\right)=(0,0)$ and given by [12]

$$
\overline{\overline{\alpha_{0}}}=\left(\begin{array}{ccc}
\frac{2\left(\epsilon_{n}-1\right)}{\epsilon_{n}+1} & 0 & 0 \\
0 & \frac{2\left(\epsilon_{n}-1\right)}{\epsilon_{n}+1} & 0 \\
0 & 0 & \epsilon_{n}-1
\end{array}\right)
$$

$\epsilon_{n}$ is the permittivity of the needle, and $\overline{\bar{T}}$ is the coordinate transformation matrix given by [13]

$$
\overline{\bar{T}}=\left(\begin{array}{ccc}
\cos \theta_{t} \cos \phi_{t} & \cos \theta_{t} \sin \phi_{t} & -\sin \theta_{t} \\
-\sin \phi_{t} & \cos \phi_{t} & 0 \\
\sin \theta_{t} \cos \phi_{t} & \sin \theta_{t} \sin \phi_{t} & \cos \theta_{t}
\end{array}\right) .
$$

Due to the azimuthal symmetry of the needles, $\phi_{t}$ is a random variable uniformly distributed over $[0,2 \pi]$. Averaging over $\phi_{t}$ leads to a diagonal tensor $\overline{\bar{\alpha}}$ with $\alpha_{11}=\alpha_{22}=\left[\left(1+\cos ^{2} \theta_{t}\right) /\left(\epsilon_{n}+1\right)+\left(\sin ^{2} \theta_{t}\right) /(2)\right]\left(\epsilon_{n}-1\right)$, and $\alpha_{33}=\left[\left(2 \sin ^{2} \theta_{t}\right) /\left(\epsilon_{n}+1\right)+\cos ^{2} \theta_{t}\right]\left(\epsilon_{n}-1\right)$.

After implementing DBA algorithm for the needle clusters, the scattering pattern is compared with the MoM exact solution. Fig. 9 shows the forward scattering from an end-cluster versus the incident angle. The DBA solution (dashed line) agrees well with the MoM solution (solid line) with less than $0.5 \mathrm{~dB}$ error magnitude and less than $10^{\circ}$ in the phase. Fig. 10 plots the bistatic scattering patterns from the same needle cluster at angles near the forward scattering direction. Comparison with MoM shows very good agreements in both magnitude and phase of the bistatic scattering patterns. In both Figs. 9 and 10, only H-polarization results are shown. Similar results are obtained for V-polarization case as well. It should be mentioned that the simulations results presented so far are all based on free space environment. The ground plane effect on the scattered wave from the needle clusters is taken into account afterwards in the wave propagation model using a geometric optics approach [2], [14]. Since the needle clusters are not very close to the ground (many wavelengths away), the ground does not affect the multiple scattering happened within the needle clusters.
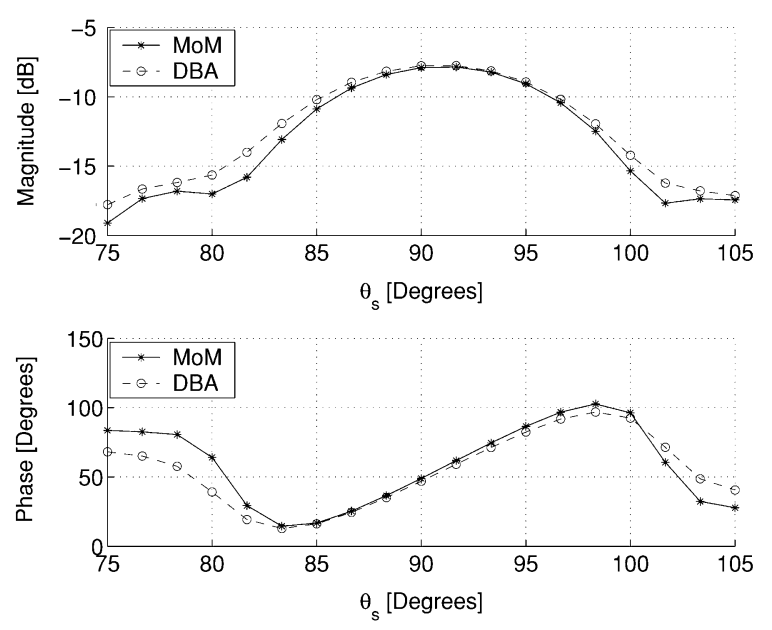

Fig. 10. Comparison of bistatic scattering pattern from a needle cluster between DBA and MoM: $S_{h h}$.

It should be noted that the DBA algorithm described above requires numerical integration over the volume of scatterer, however, compared to MoM, DBA is much less computationally intensive, and is scalable with the increase in the number of needles since the shape of the average scatterer is not affected by the number and only the needle volume fraction increases. The computation time for the end-cluster using MoM takes about $1 \mathrm{~h}$, whereas the same simulation using DBA takes only about $20 \mathrm{~s}$.

In the next section, the effectiveness of the macromodel is demonstrated by comparing simulation of wave propagation through a red pine stand with measured results.

\section{Outdoor Measurement of WaVe Propagation THROUGH Foliage}

In this section, the procedure for an outdoor wave propagation measurement is described and the comparison between the measurement and wave propagation simulation results is presented. Simulation results using single scattering and using the macromodel for needles are both presented to indicate the improvement of wave propagation model by including the effect of multiple scattering in the dense leaf clusters.

The outdoor measurement was conducted in November 2002, for a pine tree stand including 13 red pine trees ( 5 rows, $2-3$ trees each row) at $K a$-band ( $35 \mathrm{GHz}$ ). This stand occupies a $15 \mathrm{~m} \times$ $25 \mathrm{~m}$ area with average distance of $5 \mathrm{~m}$ between two adjacent trees. The average tree height, crown depth, and crown diameter are 7.5, 6.4, and $5.5 \mathrm{~m}$, respectively.

Fig. 11 shows the measurement system block diagram designed for this experiment. Power measurement was performed for determining the path-loss. The transmitted power was set at $23 \mathrm{dBm}$ which was radiated through a horn antenna with a gain of $28 \mathrm{~dB}$. The received signal was down-converted to an IF band (30 MHz) using a harmonic mixer. The IF signal was then amplified and filtered before detection by a spectrum analyzer. Receiver antenna was a horn antenna identical to the transmitter antenna having a half-power beamwidth of $10^{\circ}$. The transmitter was located in a clear area, $20 \mathrm{~m}$ away from the tree stand, illuminating it from the side. The distance was chosen so that 


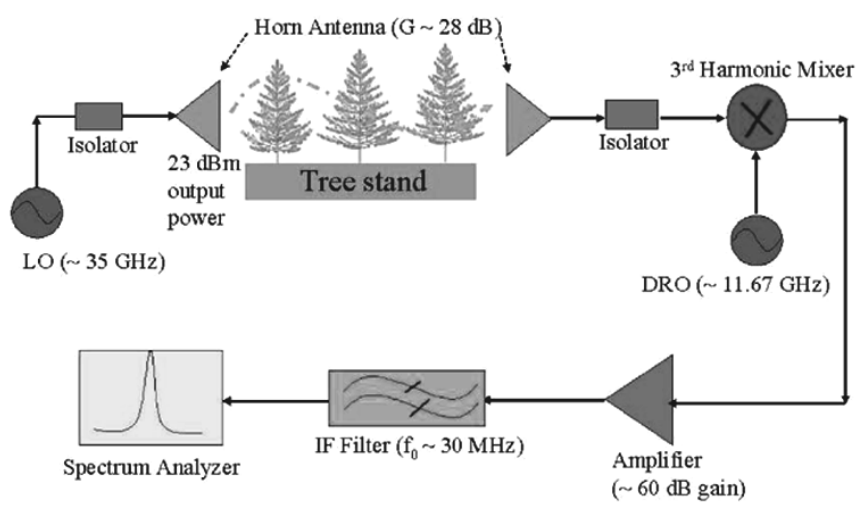

Fig. 11. Block diagram of the wave propagation measurement system.

the tree line was in the far-field of the transmitter antenna. The antenna footprint at $20 \mathrm{~m}$ away is about $3 \mathrm{~m} \times 3 \mathrm{~m}$ which is substantially smaller than the tree crown dimensions, ensuring a distributed illumination. The receiver was first set up in front of the tree line for calibrating the system, and then moved behind the trees for taking power measurements. The received power in front of the tree stand is proportional to the system gain minus the free space loss, and the power received behind the tree stand is equal to the same system gain minus the sum of a different free space loss and the path-loss due to the tree stand. By comparing these two power measurements and correcting for the difference in the free space loss, the path-loss through the tree stand is obtained.

For this measurement both the transmitter and receiver antennas were kept $1.3 \mathrm{~m}$ above the ground, at a height above the trunk layer. The alignment of the transmitter and receiver antennas was achieved by attaching a laser pointer parallel to the antenna boresight at each antenna. To acquire the desired path-loss statistics, 84 independent spatial samples of transmitted signal through the pine stand were collected. A coherent wave propagation model [3] based on Monte Carlo simulation of realistic looking fractal trees is used to simulate propagation and scattering through the tree stand. Ground truth measurements were performed to ensure that the computer-generated fractal tree model is as close as possible to the real trees. The model in [3] is modified for the normal incidence case, and the antenna patterns of both transmitter and receiver antennas are taken into account. 100 realizations were performed for this Monte Carlo simulation to obtain the statistics of the field through foliage. The cumulative distribution function (CDF) of the calibrated path-loss from outdoor measurements (solid line) and computer simulations are shown in Fig. 12. Table I also shows the mean and standard deviation of the path-loss of these results. It is obvious that single scattering applied to pine needles whose scattering are computed by Rayleigh-Gans approximation (dotted line) grossly overestimates path-loss at millimeter-wave frequencies. Using single scattering in conjunction with semi-exact solution to calculate the scattering from individual needles (dashed line), the path-loss estimate improves but there is still $8-9 \mathrm{~dB}$ of difference between the simulation and measured results. Using the macromodel where the multiple scattering effects in the needle clusters are accounted for, in the wave propagation model (dot-dash line) the

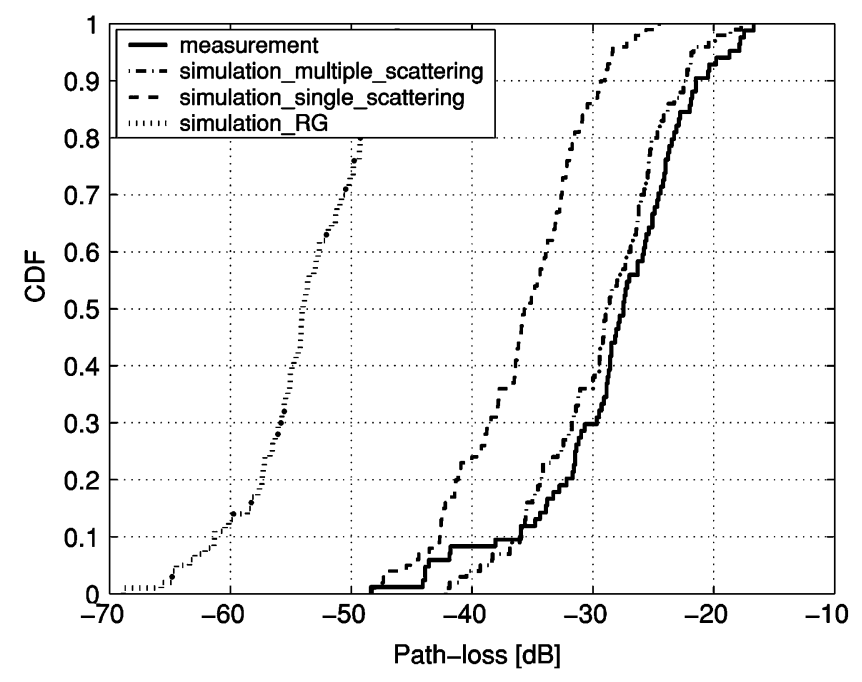

Fig. 12. Comparison of path-loss through foliage between measurement and simulation results.

TABLE I

COMParison of MEAN AND StAND DEVIATION OF PATH-Loss BetweEn MEASUREMENT AND SIMULATION RESUlTS

\begin{tabular}{c|c|c}
\hline & Mean (dB) & Std. (dB) \\
\hline Measurement & -24.8 & -23.8 \\
\hline Multi. Scat. (macro-model) & -26.4 & -25.6 \\
\hline Single Scat. (semi-exact) & -33.1 & -32.3 \\
\hline Single Scat. (Rayleigh-Gans) & -51.7 & -51.9 \\
\hline
\end{tabular}

agreement between the measurement and simulation results is markedly improved. It is also worth mentioning that the macromodel also improves the computation time of the wave propagation simulation as well. This is due to the fact that many needles $\sim 100$ ) are lumped into one scatterer, and the scattering matrix of such scatterer is pre-computed and stored to be used as a lookup table. Comparing the computation time of the propagation simulation using the macromodel multiple scattering and the Rayleigh-Gans single scattering, a speedup of about $30 \%$ is achieved (the latter one takes about $10 \mathrm{~h}$ ).

\section{CONCLUSION}

In this paper the accuracy of single scattering theory for estimation of attenuation rate of a dense medium like a needle cluster is examined by comparing methods based on single scattering theory and a full-wave solution MoM. Simulations of forward scattering show that single scattering theory usually overestimates the attenuation rate. This is severe at high frequencies since scatterers become electrically large and fall within near field of each other.

To incorporate the multiple scattering effects from needle clusters without increasing the complexity or the computation time, a novel macromodeling approach is presented. This is done by modeling the bistatic scattering outside the forward scattering beam by an incoherent field having a constant average bistatic scattering pattern, and inside the forward scattering beam by DBA. It is shown that when DBA is applied to the equivalent "average scatterer" having an inhomogeneous 
and anisotropic dielectric constant the mean bistatic scattered field can be computed very accurately. The results of macromodel for an end-cluster and a stem-cluster are compared with the MoM solution. The macromodel while being accurate is by two orders of magnitude faster than MoM solution.

An outdoor path-loss measurement was conducted for a pine tree stand to examine the accuracy of the wave propagation model using the macromodel. The comparison between the measurement and simulation results shows that for this specific case the propagation model that uses single scattering overestimates the path-loss by $8-9 \mathrm{~dB}$, whereas when the macromodel is used path-loss estimate came to within 1 to $2 \mathrm{~dB}$ of the measured results.

\section{REFERENCES}

[1] H. L. Bertoni, Radio Propagation for Modern Wireless Systems. Englewood Cliffs, NJ: Prentice-Hall, 2000.

[2] Y.-C. Lin and K. Sarabandi, "A monte carlo coherent scattering model for forest canopies using fractal-generated trees," IEEE Trans. Geosci. Remote Sensing, vol. 37, no. 1, pp. 440-451, Jan. 1999.

[3] I.-S. Koh and K. Sarabandi, "Polarimetric channel characterization of foliage for performance assessment of GPS receivers under tree canopies," IEEE Trans. Antennas Propag., vol. 50, no. 5, pp. 713-726, May 2002.

[4] L. L. Foldy, "The multiple scattering of waves," Phys. Rev., vol. 67, pp. 107-119, 1945.

[5] I.-S. Koh, F. Wang, and K. Sarabandi, "Estimation of coherent field attenuation through Dense Foliage including multiple scattering," IEEE Trans. Geosci. Remote Sensing, vol. 41, no. 5, pp. 1132-1135, May 2003.

[6] J. J. H. Wang, Generalized Moment Methods in Electromagnetics. New York: Wiley, 1991, ch. 7.

[7] R. Schiffer and K. O. Thielheim, "Light scattering by dielectric needles and disks," J. Appl. Phys., vol. 50, no. 4, pp. 2476-2483, 1979.

[8] G. T. Ruck, Radar Cross Section Handbook. New York: Plenum Press, 1970, vol. 1.

[9] D. Polder and J. H. van Santen, "The effective permeability of mixtures of solids," Physica, vol. XII, no. 5, pp. 257-271, 1946.

[10] A. Ishimaru, Wave Propagation and Scattering in Random Media. New York: IEEE Press, 1997, pp. 134-139.

[11] R. J. E. Clausius, Die Mechanische Behandlung Der Electricität, 1879. Abschnitt III (F. Vieweg, Braunschweig).

[12] K. Sarabandi and T. B. A. Senior, "Low-frequency scattering from cylindrical structures at oblique incidence," IEEE Trans. Geosci. Remote Sensing, vol. 28, no. 5, pp. 879-885, May 1990.

[13] G. B. Arfken and H. J. Weber, Mathematical Methods for Physicists, 4th ed. San Diego, CA: Academic Press, 1995.

[14] T. Chiu and K. Sarabandi, "Electromagnetic scattering from short branching vegetation," IEEE Trans. Geosci. Remote Sensing, vol. 38, no. 2, pp. 911-925, 2000.

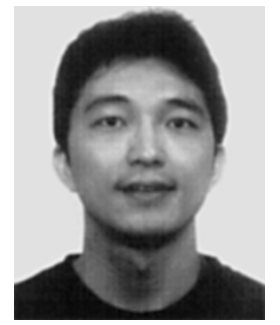

Feinian Wang (S'02) was born in China. He received the B.S. degree in physics from Tsinghua University, Beijing, China, in 1998 and the M.S. degree in electrical engineering from the University of Houston, Houston, TX, in 2000. He is currently working toward the Ph.D. degree in electrical engineering at the Radiation Laboratory at the University of Michigan, Ann Arbor.

In 2001, he worked as an RF Engineer in Comsearch, Northern Virginia. His research interests include theoretical modeling and measurements of wave propagation, numerical techniques for random media scattering, radar and Interferometry systems.

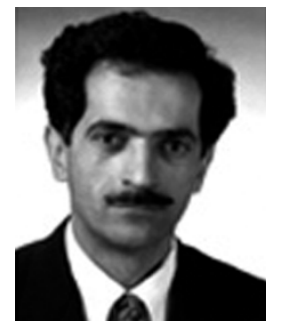

Kamal Sarabandi (S'87-M'90-SM'92-F'00) received the B.S. degree in electrical engineering from Sharif University of Technology, Tehran, Iran, in 1980, the M.S. degree in electrical engineering/mathematics, and the Ph.D. degree in electrical engineering from The University of Michigan, Ann Arbor, in 1986 and 1989, respectively.

$\mathrm{He}$ is Director of the Radiation Laboratory and a Professor in the Department of Electrical Engineering and Computer Science, The University of Michigan-Ann Arbor. He has 20 years of experience with wave propagation in random media, communication channel modeling, microwave sensors, and radar systems and is leading a large research group consisting of four research scientists, $12 \mathrm{Ph}$.D. students, and two M.S. students. Over the past ten years he has graduated $20 \mathrm{Ph}$.D. students. He has served as the Principal Investigator on many projects sponsored by NASA, JPL, ARO, ONR, ARL, NSF, DARPA, and numerous industries. He has published many book chapters and more than 115 papers in refereed journals on electromagnetic scattering, random media modeling, wave propagation, antennas, microwave measurement techniques, radar calibration, inverse scattering problems, and microwave sensors. He has also had more than 230 papers and invited presentations in many national and international conferences and symposia on similar subjects. His research areas of interest include microwave and millimeter-wave radar remote sensing, electromagnetic wave propagation, and antenna miniaturization.

Dr. Sarabandi is a Member of the International Scientific Radio Union (URSI) Commission F and of The Electromagnetic Academy. He received the Henry Russel Award from the Regent of The University of Michigan, (the highest honor the University of Michigan bestows on a faculty member at the assistant or associate level). He received a 1996 Teaching Excellence Award from the Department of Electrical Engineering and Computer Science and a 1999 GAAC Distinguished Lecturer Award from the German Federal Ministry for Education, Science, and Technology, given to about ten individuals worldwide in all areas of engineering, science, medicine, and law. He also received the 2003/2004 College of Engineering Research Excellence Award, The University of Michigan-Ann Arbor. In the past several years, joint papers presented by his students at a number of symposia (IEEE AP'95, '97, '00, '01, '03 IEEE IGARSS'99, '02, IEEE MTTS'01) have received student prize paper awards. He is a Vice President of the IEEE Geoscience and Remote Sensing Society (GRSS), a past Chairman of the Awards Committee of the IEEE GRSS from 1998 to 2002, and a Member of the IEEE Technical Activities Board Awards Committee from 2000 to 2002. He is an Associate Editor of the IEEE Transactions on Antennas and Propagation and the IEEE SENSORS JOURNAL. He is listed in American Men \& Women of Science, Who's Who in America, and Who's Who in Electromagnetics. 